\title{
Historische und pädagogische Grundlagen der Olympischen Erziehung
}

Roland Naul

\section{Abstract}

Die historischen und pädagogischen Grundlagen der Olympischen Erziehung haben verschiedene Wurzeln und Quellen. Eine dieser zentralen Grundlagen stellen die Schriften von Pierre de Coubertin dar. Seine Überlegungen konzentrieren sich auf den Sinn und Zweck der Olympischen Spiele und auf die Aufgaben und Ziele des antiken Gymnasiums als einem permanenten Zentrum zwischen den Olympischen Spielen für das körperliche, geistige und moralische Training der Jugend. Als die Olympischen Spiele nach dem 1. Weltkrieg wieder Fuß gefasst hatten, verließ Coubertin das IOC und versuchte mit Partnern aus Griechenland und Deutschland die Idee des antiken Gymnasiums als eine moderne Bildungsstätte zu realisieren. Seine Nachfolger, Freunde und Förderer konnten schließlich mit Unterstützung des IOC und des Hellenischen Olympischen Komitees das antike Gymnasium im Jahr 1961 als Internationale Olympische Akademie eröffnen.

Schon früh wurde der pädagogische Gedanke der Olympischen Idee auch anlässlich von Olympischen Spielen von den ausrichtenden Städten im Rahmen des Festprogramms berücksichtigt. Die drei Jahrzehnte zwischen den Jahren 1950 und 1980 waren entscheidend, um Programme zur Förderung der Leibeserziehung, des Jugendsports und der Sportwissenschaft im Kontext Olympischer Spiele zu entwickeln. Hier liegen die pädagogischen Vorboten einer Olympischen Erziehung (Krüger, 2018), die in Deutschland erst seit den 1990er Jahren eine schrittweise Verbreitung gefunden hat.

\section{Einleitung}

Die historischen und pädagogischen Grundlagen der Olympischen Erziehung sind in einen vielschichtigen kulturhistorischen und bildungsgeschichtlichen Zusammenhang eingebettet und unterlagen dem bestän- 
digen Wandel der Olympischen Spiele ebenso wie der Entwicklung von Sportorganisationen. Auch der Olympische Kongress von Paris 1894 und der an die Spiele von Athen 1896 anschließende Olympische Kongress von Le Havre im Jahr 1897 waren als Ausgangspunkte der Olympischen Bewegung für die Entwicklung einer Olympischen Erziehung von besonderer Bedeutung. Allerdings können die philosophischen und anthropologischen Grundlagen sowie die Ziele einer Olympischen Erziehung durchaus noch weiter zurückdatiert werden. Die pädagogischen Grundlagen sind in den anthropologischen Schriften von Johann Christoph Friedrich GutsMuths mit seiner pädagogischen Gymnastik gegen Ende des 18. Jahrhunderts ebenso verwurzelt wie in der christlichen Erziehungslehre von Reverend Thomas Arnold an der Public School in Rugby im frühen 19. Jahrhundert, die eine Moralerziehung durch Bewegung und Sport einschloss (Naul, 2010).

Dieser Beitrag stellt die historischen und pädagogischen Grundlagen der Olympischen Erziehung, eingeleitet durch Pierre de Coubertin, in den Vordergrund. Im Zentrum stehen seine Aktivitäten und Publikationen in den ersten beiden Dekaden des 20. Jahrhunderts sowie seine berühmte Radiorede aus dem Jahr 1935 über die fünf Olympischen Prinzipien.

Hervorgehoben werden auch die Leistungen seiner Mitstreiter im 1. Internationalen Olympischen Komitee (IOC), die wegen der Fokussierung auf die Person Pierre de Coubertins, über viele Jahre nur wenig Beachtung fanden. Einige von ihnen entwickelten als Lehrer und Offiziere viele eigene Ideen, wie Schulen die Olympische Idee aufgreifen und entwickeln können. Bereits sehr früh gab es Vorschläge zur Rolle der Schule in der Olympischen Bewegung, die zeitnah auch in deutscher Sprache publiziert wurden. Von Pierre de Coubertin selbst wurden diese Ideen inhaltlich erst viele Jahre später noch einmal spezifisch artikuliert, nachdem er sein Amt als Präsident des IOC niedergelegt hatte (1925).

Ein Kerngedanke dieses Artikels ist auch die Idee des antiken Gymnasiums und Coubertins zahlreiche Versuche, ab dem Ende der 1920er Jahre ein modernes Trainingszentrum für eine geistige und körperliche Erziehung zum Zwecke einer harmonischen Gesamtbildung zu errichten, lange bevor in diesem Sinne im Jahr 1961 die Internationale Olympische Akademie (IOA) im antiken Olympia eröffnet wurde.

Nicht zuletzt stellt die Entwicklung der Olympischen Spiele in den letzten 125 Jahren ebenfalls einen historischen Vektor für die Entwicklung der Olympischen Erziehung, ihrer pädagogischen Ideale, Ziele und Inhalte dar. Inwieweit diese Entwicklung durch das Kultur- und Wissenschaftsprogramm der Gastgeberstädte von Olympischen Spielen beeinflusst oder geprägt wurde, ist ein weiterer Teil der folgenden Ausführungen. Einen 
besonderen Wendepunkt stellt das Jahr 1983 dar, als das IOC alle Nationalen Olympischen Komitees aufforderte, so noch nicht geschehen, eigene Nationale Olympische Akademien zu gründen, um die Olympische Erziehung nachhaltig zu fördern.

\section{Die Ideen Pierre de Coubertins über Erziehung}

\section{Pierre de Coubertin}

Pierre de Frédy, Baron de Coubertin (1863 - 1937) war ein französischer Pädagoge, Sportfunktionär und Historiker. Er studierte an der Sorbonne Kunst, Philologie und Rechtswissenschaften und wollte durch eine neue Form der sportlichen Erziehung den ganzen Menschen in der Einheit von Körper, Geist und Seele formen. Er ist verantwortlich für Einführung der Olympischen Spiele der Neuzeit und gründete 1894 das Internationale Olympische Komitee. Coubertin entwarf die Olympischen Ringe und war der erste Olympiasieger in der Disziplin Literatur.

Die moderne Wiedererweckung der Olympischen Spiele am Ende des 19. Jahrhunderts war vorrangig ein pädagogisches Projekt. Pierre de Coubertin, ein französischer Schulreformer, forderte in den späten 1880er Jahren mit einigen Gleichgesinnten die Reform der militärischen Schulgymnastik an den höheren Schulen Frankreichs durch die Einführung athletischer und sportlicher Übungen, die er selbst bei Besuchen in englischen Public Schools kennengelernt hatte. Coubertins Erziehungsidee fußte dabei vor allem auf zwei Grundlagen: der griechischen, antiken Bildungsidee einer harmonischen Erziehung von Körper und Geist sowie der körperlichen Erziehung an den englischen Public Schools als eine ganzheitliche Charakterschulung.

Für Coubertin war das antike Bildungsideal „a delicate balance between mind and body" grundlegend für seine Reformpläne (Coubertin, 1894, in Müller, 2000, S. 532). Ebenso wurde er von den pädagogischen Ideen eines Thomas Arnold (1795-1842) beeinflusst, der als Head Master der Public School von Rugby die Durchführung körperlicher Übungen erweitert hatte. Coubertin besuchte außerdem auf mehreren Reisen in den 1880er Jahren die Eliteschulen Englands und der USA und beobachtete, mit welcher Begeisterung Schüler und Studenten Mannschaftsspiele (cricket, football, baseball) als Teil des offiziellen Curriculums betrieben, die die charakterliche Bildung fördern sollten (Mac Aloon, 2007; Wassong, 2002). In dieser Hinsicht finden Coubertins olympische Aktivitäten und Publika- 
tionen ihren Ursprung in den 1880er Jahren, lange bevor er die Idee des Olympischen Kongresses 1894 an der Sorbonne in Paris umsetzte und die ersten internationalen Olympischen Spiele organisiert wurden.

In seinen frühen Bemühungen, sein Erziehungsideal zu beschreiben, griff Coubertin auf zwei für ihn zentrale Begriffe zurück: den „cult of effort" und den "cult of eurythmy“. "Cult of effort" kann als das ständige Bestreben nach der Verbesserung der eigenen Fähigkeiten und Leistungen umschrieben werden. "Cult of eurythmy“ beschreibt das Gleichmaß oder den Gleichklang von körperlicher, geistiger, kultureller bzw. künstlerischer Bildung. Das nachfolgende Zitat Coubertins stammt aus seinen Olympischen Briefen des Jahres 1918, die er schrieb, nachdem das Büro des IOC während des Ersten Weltkriegs von Paris nach Lausanne verlegt worden war:

"This Olympic pedagogy which I recently said was based at once on the cult of effort combined on the cult of eurhythmy - and consequently on the love of excess combined with the love of moderation - is not sufficiently served by being glorified before the world once every four years in the Olympic Games. It needs permanent factories. The Olympic factory for the ancient world was the gymnasium. The Olympiads have been renewed, but the gymnasium of antiquity has not - as yet. It must be" (Olympischer Brief No. V, Coubertin, 1918, in Müller, 2000, S. 217).

Coubertin entlieh den Begriff Eurythmie von Erziehungsansätzen wie denen Rudolf Steiners in den frühen Jahren nach 1900, der z.B. in Deutschland mit reformpädagogischen Tendenzen in der Kunsterzieherbewegung einherging. Der Begriff unterstreicht eine Pädagogik, die auf eine Harmonie von Bewegungsvorstellung und künstlerischem Ausdruck ausgerichtet ist und eine intellektuelle, praktische und künstlerische Gestaltung in sich vereint.

Es ist nicht schwer zu verstehen, warum eine solche philosophischphilanthropische Strömung einen Pädagogen wie Coubertin beeindruckte, der das französische Erziehungssystem aufgrund seiner reinen Wissensvermittlung scharf kritisierte. Analog zur Wiedererweckung der Olympischen Spiele war Coubertin daher zusätzlich daran interessiert, das Gymnasium der Antike wieder ins Leben zu rufen. Seine Vorstellung der Wiedereinführung des Gymnasiums der Antike beinhaltete die Einrichtung einer Kulturstätte, ausgelegt auf die Ausgewogenheit von körperlichem Training, geistiger Bildung und Charakterschulung für eine harmonische und ganzheitliche Erziehung junger Menschen. 
In dem oben zitierten Olympischen Brief nannte Coubertin „städtische Institutionen“ als eine bedeutende Möglichkeit, solche Kulturfabriken dauerhaft zu installieren (Müller, 2000). In der Tat entstanden in den 1920er Jahren in den Städten vieler europäischer Länder Sportschulen und Sportvereine, die dauerhaft körperliche Übungen und eine sportliche Erziehung förderten. Seitdem wird zwar nicht an jedem dieser Orte und Sportstätten der Eurythmie entsprochen, jedoch zumindest der cult of effort für die Jugend mit Training und Wettkampf gefördert. Effort oder Anstrengung wurde ein fundamentales Olympisches Prinzip, heute bekannter als joy found in effort. Ebenso gibt es den Begriff der Eurythmie als Thema und Referenzpunkt in olympischen Studien bis heute. Für Coubertin sind beide Prinzipien seit den 1920er Jahren zentrale Pfeiler seiner "pedagogie sportive" (Coubertin, 1922), die wir heute essenziell mit einer Olympischen Erziehung verbinden.

Coubertin selbst sprach in Bezug auf seine pädagogischen Reformen nie von einer Olympischen Erziehung. Er bevorzugte Begriffe wie „athletische Erziehung“, „englische Erziehung“ oder „pedagogie sportive“, wie der Titel seines berühmten Buches aus dem Jahr 1922 heißt (Coubertin, 1922). Coubertin sah wie seine Kollegen aus dem IOC in den Olympischen Spielen eine Möglichkeit zur Förderung von Olympischen Idealen und Verhaltensweisen bei den teilnehmenden Athleten. Die sportpädagogischen Reformen, die er in Unterricht und Schule verfolgte, basierten auf der Idee der Einheit von Körper und Geist bei jungen Menschen mit dem Ziel der individuellen Selbst-Verbesserung durch die Teilnahme am und die Anstrengung im Sport. Fair Play, Freundschaft, Frieden und internationale Verständigung gehörten zu der Liste der Werte, die im Konzept von Coubertins Olympismus wiederzufinden sind.

Für Pierre de Coubertin und seine Mitstreiter waren die Olympischen Spiele folgerichtig nicht nur eine beliebige Sportveranstaltung, sondern der Ausdruck einer breiten sozialen Bewegung, die durch Spiel und Sport die Entwicklung einer humanen Welt fördern sollte. Coubertins Enthusiasmus galt im Kern der Erziehung durch den olympischen Sport. Diesen sah er jedoch im Lauf der Entwicklung der Olympischen Spiele nicht genügend verwirklicht. Ein Hauptmotiv seines Rücktritts vom Amt des Präsidenten des IOC in Prag (1925) war, dass er diesen Erziehungsgedanken auch außerhalb der Olympischen Bewegung weiterverfolgen wollte, um die "unfinished pedagogical legacy of the gymnasium of antiquity", den zweiten Teil seiner Erziehungsidee, in die Tat umzusetzen. Dafür gründete er die Union pédagogique universelle. In seiner Prager Abschiedsrede beklagte er, dass man wohl nicht voll und ganz verstanden hatte, was er mit der Wiedereinführung der Olympischen Spiele erreichen 
wollte. Im IOC gab es nämlich in diesen Jahren wenig Interesse an den pädagogischen Zielen der Spiele und der Wiederbelebung der Idee des antiken Gymnasiums (Coubertin, 1922).

Pierre de Coubertin entwickelte selbst kein eigenes Konzept oder eine Systematik einer Olympischen Erziehung. In seinem Olympischen Brief No. IV erklärte er jedoch, was er unter Olympismus verstand:

"Olympism is not a system, it is a state of mind. The most widely divergent approaches can be accommodated in it, and no race or time can hold an exclusive monopoly on it" (Coubertin, 1918, in Müller, 2000, S. 548).

Wenn der Olympismus in den Augen Coubertins kein streng philosophisch oder pädagogisch begründetes oder abgeleitetes System von Axiomen und Sätzen darstellt, sondern eher eine Geisteshaltung, dann ist es nicht überraschend, dass er kein theoretisches System einer Olympischen Pädagogik entworfen hat. Er rezipierte viel mehr verschiedene pädagogische Strömungen und sportliche Orientierungen seiner Zeit und stützte so seine eigenen Vorstellungen und Ideen. Dennoch waren bereits bis Mitte der 1920er Jahre in einer Reihe von europäischen Ländern (auch in Deutschland) Erziehungs- und Schulreformen auf den Weg gebracht worden, die neue Elemente mit Spiel und Sport in den Schulalltag eingeführt hatten, auch teilweise bereits verbunden mit dem Ziel einer ethisch-moralischen Charakterbildung. Allerdings bezeichnete in dieser Zeit niemand die Förderung des Jugendsports in Schulen und Sportvereinen in Anlehnung an Coubertin als Olympische Pädagogik oder Olympische Erziehung.

Nach rund 120 Jahren der modernen olympischen Geschichte kann man feststellen, dass der Olympismus heute ein pädagogisches Konzept darstellt, das sehr verschiedene, auch divergente Ansätze, umfasst. Gleichwohl lassen sich aber in den Schriften Coubertins klare und eindeutige Aufgaben festhalten, die er in seinen zahlreichen Reden und Briefen als Erziehungsaufgabe zur Förderung des Olympischen Geistes artikuliert hat. So beschreibt er in seinem Olympischen Brief No. III vier konkrete Pflichten für Erzieher, um eine harmonische Bildung von Körper und Geist zu erreichen:

"[...] to distinguish $[\ldots]$ only the body and the mind, $[\ldots]$ is too simplistic, but rather the muscles, the understanding, the character, and the conscience. This corresponds to the four-fold duty of the educator" (Coubertin, 1918, in Müller, 2000, S. 547). 
Somit verlangt er eine Erziehung, die den Prinzipien der Eurhythmie in seinem Sinne folgt. Er wünscht sich nicht nur eine integrative Entwicklung von Körper und Geist, sondern eine Erziehung in vier Kompetenzbereichen: körperlich, sozial, moralisch und geistig.

Im Jahre 1935, kurz vor den Olympischen Spielen in Berlin, hielt Coubertin seine berühmte Radiorede, die von allen Rundfunksendern im Deutschen Reich aus der Schweiz übertragen wurde. Darin formulierte und erläuterte er fünf Prinzipien als pädagogisch-philosophische Grundlagen des Olympismus (Coubertin, 1918, in Müller, 2000, S. 580-583).

Das erste und für Coubertin wohl wichtigste olympische Prinzip ist die „religio athletae“. Er sah in den Teilnehmern an den Olympischen Spielen „Botschafter einer modernen Erziehung“ von zivilisierten Völkern, die den religiösen Geist des Sports im Sinne eines moralisch korrekten Verhaltens untereinander teilten. Athleten sollten die Repräsentanten einer „neuen humanen Gesellschaft" sein (Coubertin, 1918, in Müller, 2000, S. 580).

Das zweite Prinzip basiert auf der Gleichheit aller Menschen. Alle Athleten streben fortwährend nach der Vervollkommnung ihrer Fähigkeiten - körperlich, intellektuell und moralisch - als individuelle Selbsterfüllung im Rahmen ihrer persönlichen, holistischen und harmonischen Erziehung. Diese Athleten waren in den Augen von Coubertin „Kosmopoliten“, welche die Elite ihres jeweiligen Landes darstellten, da sie physische Überlegenheit und Vielseitigkeit zum Ausdruck brachten. In ihrer Entwicklung folgten sie dem Wahlspruch „citius, altius, fortius“ (schneller, höher, stärker) (Coubertin, in Müller, 2000, S. 581). Hier meint Coubertin nicht die dauerhafte Steigerung von Höchstleistungen, sondern die Verpflichtung eines jeden Athleten, seine individuellen Leistungen beständig zu verbessern.

Das dritte Prinzip ist mit dem von Coubertin assimilierten Konzept der „Ritterlichkeit“" verbunden. Coubertin sieht im Kampf der Athleten eine Parallele zu den Rittern in alten Tagen, die gegeneinander unter rigiden Regeln der Ehre und auf der Grundlage gegenseitigen Respekts („respect mutuelle“) gekämpft haben. Er schreibt dazu:

"In chivalry, the idea of competition, of effort opposing effort for the love of the effort itself, of courteous yet violent struggle, is superimposed on the notion of mutual assistance, the basis of camaraderie" (Coubertin, 1918, in Müller, 2000, S. 581).

An anderen Stellen seiner Schriften hat Coubertin auch von Fair Play gesprochen, wenn er Ritterlichkeit meinte und von joy in effort anstelle von Kraftanstrengung. Beide Begriffe sind heute zwei der wichtigsten Prinzipien einer Olympischen Erziehung. 
Das vierte Prinzip des Olympismus beschreibt Coubertin als truce, als Gottesschutz oder auch Waffenstillstand. Die Idee der rhythmischen Wiederkehr von Olympischen Spielen war gemäß dem antiken Vorbild mit einer Immunität aller Teilnehmer, die eine sichere Reise zu den Spielen und zurück garantierte, verbunden (Lämmer, 1982/83). Die regelmäßige Durchführung von Olympischen Spielen in einem Rhythmus von vier Jahren war für Coubertin von großer Bedeutung. Er hoffte, dass die Durchführung der Spiele zu einer Unterbrechung von Konflikten und Kriegen zwischen den Völkern beitragen würde. Der Rhythmus von Olympischen Spielen könne die Feindseligkeiten, Dispute und Missverständnisse zwischen den Völkern zumindest zeitweilig unterbrechen.

Das fünfte und letzte Prinzip ist in der Idee der Eurhythmie verankert. Coubertin wollte damit Fest und Feier, Kunst, Musik, Literatur und Theater als Elemente in die Inszenierung von Olympischen Spielen einbeziehen. Von den Stockholmer Spielen 1912 bis zu denen in London 1948 gehörten sogar Kunstwettbewerbe zum Olympischen Wettkampfprogramm.

Coubertins Erziehungsideen und Vorstellungen von der Entwicklung des olympischen Gedankenguts können somit als eine Mischung aus verschiedenen Begriffen, geistigen Strömungen und Wertvorstellungen der Zeit um 1900 angesehen werden. Dies ist unter anderem an seinen Vorstellungen über die eurythmische Entwicklung von Athleten, über ihre Rolle als Botschafter der Erziehung ihres Landes und in der Bedeutung der Olympischen Spiele mit ihrem Festprogramm für Gesellschaft und Frieden wieder zu erkennen.

Coubertins Ausführungen zum Olympismus und seine Vorstellungen über den Beitrag des olympischen Sports für die Erziehung der Jugend sind oft kritisiert worden, weil sie in sich kein klares, geordnetes System nach philosophischen Grundsätzen oder pädagogischen Maximen darstellen. Mit dem Zitat von Coubertin über den Olympismus ,as a state of mind" kann geantwortet werden, dass er dieses Ziel selbst nie angestrebt hat. Denn Coubertin sah den Olympismus nicht als ein philosophisches System im Aristotelischen Sinne, sondern als eine zeitgeschichtlich angewandte Form einer geistigen Haltung, die sich in verschiedenen Epochen der Zeitgeschichte weiterentwickelt und auch in anderen Kulturräumen zu neuen Adaptionen des Olympischen Gedankens führen kann. Diese zeitgeschichtlich und kulturgeschichtlich offene, flexible und sich auch ändernde Form des Olympismus macht die eigentliche Leistung im Denken Coubertins aus: „The most widely divergent approaches can be accommodated in it, and no race or time can hold an exclusive monopoly on it" (Coubertin, in Müller, 2000, S. 548). Deshalb ist die vermeintliche 
Schwäche des Gedankengebäudes von Coubertin über den Olympismus zugleich seine Stärke über Kulturräume und Zeiten hinweg.

\section{Die Mitglieder des ersten Internationalen Olympischen Komitees als Erzieher}

\section{Olympische Charta}

Die Olympische Charta beinhaltet die grundlegenden Prinzipien des Olympismus, Bestimmungen der Olympischen Bewegung sowie Regeln für die Organisation von Olympischen Spielen. Die Charta wird vom IOC herausgegeben und regelmäßig aktualisiert. Wichtige Ziele der Olympischen Charta sind die Bestimmung und Bewahrung olympischer Werte und Prinzipien.

Die erste Fassung der Olympischen Charta wurde von Coubertin und den Mitgliedern des 1. Internationalen Olympischen Komitees verfasst, aber erst 1908 als Annuaire, Règlement publiziert (COI, 1908). Erst Jahre später wurden vier zentrale Ziele in diese Charta aufgenommen:

1. Die Förderung von Verhaltensweisen, auf deren Grundlage im Sinne der Olympischen Werte Sport getrieben werden soll.

2. Die Erziehung der Jugend durch den Sport im Geiste von Verständigung und Freundschaft, um so zu einer besseren und friedlicheren Welt beizutragen.

3. Die weltweite Verbreitung der Olympischen Prinzipien und damit die Herstellung von internationalem Verständnis füreinander.

4. Die Zusammenführung der Athleten der Welt zu einem alle vier Jahre stattfindenden großen Sportfest, den Olympischen Spielen.

Als Pierre de Coubertin seine Initiative zur Wiedererweckung der Olympischen Spiele begann, sammelte er um sich herum Persönlichkeiten wie den böhmischen Gymnasiallehrer Jiri Guth-Jarkovsky, den ungarischen Pädagogen und Vertreter der Friedensbewegung Ferenc Kemeny, oder Viktor Balck und Alexander Butovsky, die in Stockholm bzw. St. Petersburg mit der körperlichen Ausbildung von Gymnastiklehrern und Offizieren beschäftigt waren. Dazu kam der Grieche Dimitrios Vikelas, der sich, wie Coubertin in Frankreich, in seinem Heimatland als eifriger Förderer der Volks- und Jugendbildung Verdienste errungen hatte. Nur eine Person aus dem ersten Komitee war beruflich nicht in der Lehre oder Ausbildung von Jugendlichen, Gymnastiklehrern oder Offizieren tätig: der Chemiker Dr. Willibald Gebhardt, der als Wissenschaftler und Autor zur körperlichen Erziehung der Schuljugend Stellung bezog (Hamer, 1971; 
Huhn, 1992; Naul 1999). Diese Männer können als Väter der Olympischen Erziehung in ihren Heimatländern angesehen werden.

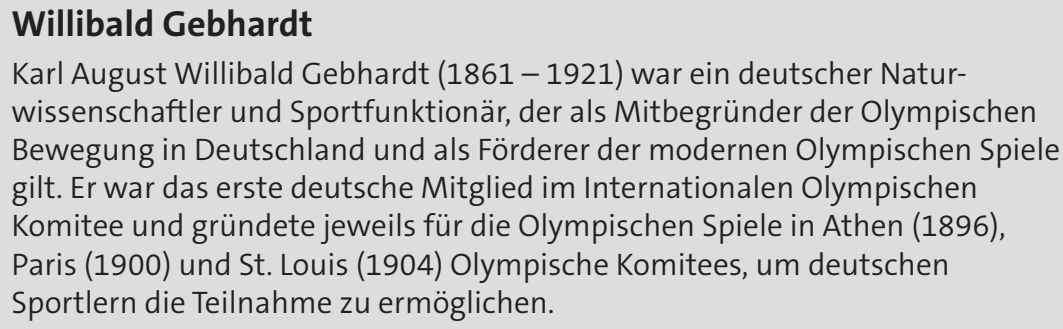

Die Olympischen Spiele von Athen 1896 hatten bei den IOC-Mitgliedern einen nachhaltigen Eindruck hinterlassen, besonders bei den Pädagogen. Zuhause berichteten Jiri Guth-Jarkovsky (1896) und Ferenc Kemeny (1897) in Zeitschriftenartikeln über die Athener Spiele und wollten die olympischen Impulse, die sie erfahren hatten, für ihre Arbeit an den Schulen umsetzen. Englischer Sport sollte stärker gefördert, Wettkämpfe und Turniere sollten organisiert und durchgeführt werden. Kemeny ging mit seinen Vorschlägen sogar so weit, dass die besten Schüler an nationalen Olympischen Spielen teilnehmen sollten (Naul, 1999).

Im März 1904 setzte Dr. Willibald Gebhardt als erstes deutsches IOCMitglied einen wichtigen Meilenstein für die Olympische Erziehung an deutschen Schulen. Er gründete zunächst zusammen mit seinen früheren Gegnern aus der Deutschen Turnerschaft (DT) und dem Zentralausschuss für Volks- und Jugendspiele (ZA) das erste, ständige Nationale Olympische Komitee, den Deutschen Reichsausschuss für Olympische Spiele (DRAfOS). Nur einen Monat später und nur wenige Tage vor seiner Abreise zu den Olympischen Spielen von St. Louis als Delegationsleiter und Repräsentant des IOC hielt er beim 1. Internationalen Kongress zur Schulhygiene in Nürnberg einen Vortrag zum Thema „Olympische Bewegung und die Schule“ (Gebhardt, 1904). Darin kritisierte er, dass die Mehrzahl der akademisch gebildeten Menschen nie den erzieherischen und moralischen Nutzen von Turnen, Spiel und Sport anerkannt hätten. Er argumentierte, „daß die Pflege des Körpers für die Bildung des Charakters von größter Notwendigkeit“ sei, um „Mut, Selbstvertrauen, Mäßigkeit, Beherrschung der Leidenschaften und sonstige Mannestugenden“ (Gebhardt, 1904, S. 109) zu entwickeln. 
In diesem Zusammenhang nannte Gebhardt drei zentrale Wesensmerkmale einer Olympischen Erziehung, ohne diesen Begriff dafür schon zu benutzen:

1. die "soziale Bedeutung der Leibesübungen", die "ausgleichend und versöhnend" auf die einzelnen Personen wirken;

2. die "nationalen Werte der Leibeskultur für die Wehrfähigkeit";

3. die internationale Funktion für die Zusammenführung der verschiedenen Völker, indem nationale Vorurteile zwischen ihnen abgebaut würden und dem Chauvinismus vorgebeugt werde (Gebhardt, 1904, S. 109f.).

Gebhardt machte auch deutlich, wie der neu gegründete DRAfOS sich eine derartige Erziehung an Schulen vorstellte:

„Unsere Organisation will vor allem dazu beitragen, das bestehende Unterrichts- und Erziehungssystem reformieren zu helfen. Die sogenannte 'wissenschaftliche Erziehung' soll aus ihrer herrschenden Stellung verdrängt, die körperliche Erziehung ihr gleichberechtigt an die Seite gestellt werden" (Gebhardt, 1904, S. 113).

Diese Gleichberechtigung bezeichnet Gebhardt als eine harmonische Erziehung, wie sie das Ideal des antiken griechischen Gymnasiums gewesen sei (Gebhardt, 1904, S. 114).

Gebhardt sah ebenso wie Coubertin in den modernen Public Schools in England das Vorbild, um mit sportlichen Übungen in Deutschland eine männliche Charaktererziehung zu verwirklichen. Dafür formulierte er am Ende seines Vortrags fünf Leitlinien:

1. Der Nachmittagsunterricht und die Hausaufgaben sind abzuschaffen; das freie Spielen fülle die Nachmittage aus. Die wissenschaftlichen Lehrer sollten angeregt werden, sich an diesen Spielen zu beteiligen, sie eventuell zu leiten.

2. Die Examina sollten auf das äußerste Maß beschränkt werden; in ibnen muß gleich dem Wissen auch das körperliche Können gewertet werden .

3. Der wissenschaftliche Unterricht ist so viel wie möglich ins Freie zu verlegen, die griechischen Peripatetiker sollten uns hierbei vorbildlich sein.

4. Die Schüler müssen mindestens alljährlich auf ihre körperliche Entwicklung und ibre Vitalkapazität untersucht, das Herz geprüft werden.

5. Es ist Unterricht in den Grundlagen der Hygiene zu erteilen. So besonders bezüglich des Einflusses von Licht und Luft, der körperlichen Reinlichkeit auf den Organismus, dann des Nutzens der Leibesübungen und der richtig angewandten Ruhe; ferner der großen Schädigungen, die durch alkoholische Getränke, durch Tabak etc. besonders auf den jugendlichen Organismus, 
hervorgerufen werden. Die Ernährung in qualitativer und quantitativer Hinsicht sollte einen wichtigen Teil dieses Unterrichts bilden (Gebhardt, 1904, S. 116).

Gebhardts Forderung der Schulreform im Kontext der Olympischen Bewegung reflektiert Kriterien und ein Wertesystem, das zu seiner Zeit nicht zum Gemeingut aller seiner Mitstreiter im IOC zählte. In dieser Zeit ging Willibald Gebhardt mit seinem detaillierten Wissen über die frühe amerikanische Hygienebewegung einen Schritt weiter als seine Zeitgenossen. Während er gleichzeitig mit seinen Kollegen im IOC die pädagogischen Werte des Sports vertrat, erweiterte er die Charakterbildung im olympischen Sinne durch die Idee einer Gesundheitsförderung mit einem aktiven Lebensstil.

Mit seinen fünf Leitsätzen dürfen wir Gebhardt als Vordenker einer Schulreform hin zu einer modernen Olympischen Erziehung einschätzen. Das gilt besonders für seine Empfehlung, die geistige und körperliche Erziehung als gleichberechtigte Bildungsziele anzusehen und in den wissenschaftlichen Unterricht Themen wie Ernährung und Hygiene einzubeziehen. Mit seinen Reformideen ist Willibald Gebhardt ein Pionier der Olympischen Erziehung in Deutschland.

Einen gewissen Effekt hatten die Ideen von Gebhardt in Deutschland bereits 1912, als die Olympischen Spiele 1916 nach Berlin vergeben wurden. Im Jahr 1913 wurde Carl Diem vom DRAfOS als Generalsekretär für die Vorbereitung dieser Spiele berufen. Primaner der Gymnasien und andere gleichaltrige Schüler an höheren Schulen sollten sich in Sichtungslehrgängen in leichtathletischen Übungen messen, um einen Olympiakader zu bilden. Mit staatlicher Unterstützung des damaligen Preußischen Kultusministeriums wurden solche Tests im Sommer 1914 erstmals durchgeführt, kurz vor dem Beginn des Ersten Weltkriegs (Lennartz, 1978).

Das pädagogische Erbe Coubertins nach seinem Rücktritt als Präsident des IOC

Nur wenige Jahre nach dem Erscheinen seiner Olympischen Briefe in der Lausanner Zeitung „La Gazette“, veröffentlichte Coubertin sein berühmtes Buch „La pedagogie sportive“ (1922), in dem er die charakterprägenden Werte und Verhaltensweisen aus Spiel und Sport als eine moderne Erziehungsaufgabe darstellte, die wir heute Olympische Erziehung nennen würden. Sein Buch wurde auch in Deutschland unter dem Titel "Sportliche Erziehung"veröffentlicht (Coubertin, 1928). Eigentlich wäre die 
richtige Übersetzung „Sportpädagogik“ gewesen, aber dieser Begriff war in den Jahren der Weimarer Republik noch nicht üblich.

Seit dem ersten Olympischen Kongress 1894 bis zu seinem Rücktritt als Präsident des IOC 1925 unternahm Coubertin immer wieder Anstrengungen, die IOC-Mitglieder von der besonderen Rolle der körperlichen Erziehung zu überzeugen. In den Jahren zwischen 1894 und 1926 organisierte Coubertin acht der zwölf bis 1994 stattfindenden Olympischen Kongresse, um die erzieherische Bedeutung des Sports aus seiner Sicht zu fördern (Müller, 1994, S. 13). Schlussendlich schreibt er:

"Preoccupied with its ever-growing technical function, the I.O.C. is unable to continue the educational task deriving from the 1897 and 1913 congresses [...]" (aus einer Rede von Coubertin, in Müller, 1994, S. 128).

Nach seinem Rücktritt in Prag 1925 widmete er sein Interesse der Gründung der Union Pedagogie Universelle und dem Bureau Internationale de Pedagogie Sportive (B.I.P.S.), um die Bedeutung der Erziehung und des Jugendsports mit den Olympischen Prinzipien in den Vordergrund zu rücken (Krüger, 2009). In dieser Zeit verfolgte Coubertin auch seine Idee der Wiedererweckung des antiken Gymnasiums verstärkt weiter. Auf einer Reise nach Griechenland im Jahr 1927 gelang es ihm, dafür das Interesse von Professor Ioannis Chrissafis, dem Leiter der Abteilung Leibeserziehung an der Universität Athen, zu wecken. Beide hatten die Idee, an antiker Stätte in Olympia ein solches Gymnasium zu errichten. Aber Chrissafis starb im Jahr 1930, bevor ihr gemeinsamer Plan realisiert werden konnte. Auch der deutsche Sportorganisator Carl Diem wurde gemeinsam mit seinem griechischen Freund Ioannis Ketseas aktiv, um eine solches Gymnasium als eine Internationale Olympische Akademie zu gründen.

Nach den Olympischen Spielen von 1936 in Berlin konnte Carl Diem als Generalsekretär des Organisationskomitees die Reichsregierung überzeugen, ein Internationales Olympisches Institut zu gründen. Diem war schon vor dem Ersten Weltkrieg ein leidenschaftlicher Verehrer von Coubertin. Im Jahr 1938 wurde dieses Institut unter seiner Leitung in Berlin eröffnet. Dort lagerten Schriften und Bücher von Coubertin, die dieser noch vor seinem Tod dem Institut übereignet hatte. Bei einem Bombenangriff im Jahr 1943 wurde der größte Teil der Bestände zerstört.

Diems Kontakte zu seinem Freund Ketseas überdauerten die Zeit bis in die 1950er Jahre. Mit ihm gemeinsam nahm er den Plan der Gründung eines Zentrums für körperliche und geistige Erziehung, ganz im Sinne von Pierre de Coubertins, nach dem Krieg wieder auf (Georgiadis, 1995, 
Müller 1995). Im Jahr 1949 konnte Ketseas das IOC überzeugen, die Gründung einer Internationalen Olympischen Akademie (IOA) zu unterstützen. Finanziert und organisiert werden sollte sie vom Hellenischen Olympischen Komitee. Es dauerte jedoch noch einmal fast ein Jahrzehnt, bis die IOA ihre 1. Session in einem Zeltlager nahe den antiken Stätten von Olympia durchführen konnte.

\section{Kultur-, Kunst- und Wissenschaftsprogramme Olympischer Spiele als Förderung einer Olympischen Erziehung}

Wie erwähnt wurden Prinzipien der körperlichen und moralischen Erziehung schon im Rahmen der frühen Olympischen Spiele vor dem 1. Weltkrieg angesprochen. Auch wurde bei der Vorbereitung und Feier von Olympischen Spielen der Erziehungsgedanke für Kinder und Jugendliche berücksichtigt. So wurde bereits bei den Olympischen Spielen in Stockholm 1912 ein Zeltlager mit 1.500 Pfadfindern durch König Gustav von Schweden initiiert. Anlässlich der Olympischen Spiele von Berlin 1936 wurde ein Internationales Sportstudentenlager mit mehr als 800 Teilnehmern durchgeführt.

Am Eröffnungstag der Spiele, am Sonntag, dem 1. August, zeigten rund 100.000 Berliner Schulkinder auf den städtischen Spiel- und Sportplätzen ihre Übungen. Zahlreiche Gruppen der Hitlerjugend marschierten durch die Straßen und nahmen an der Eröffnung der Olympischen Spiele teil. Am Sonntag, dem 9. August, dem "Marathon-Sonntag", zeigten 20.000 Berliner Jungen und Mädchen auf dem Maifeld hinter dem Olympiastadion Frei- und Ordnungsübungen („Berlins Schuljugend bei den Olympischen Spielen, Politische Leibeserziehung, 1936 (8), S.4). Außerdem gab es ein internationales Olympisches Jugendzeltlager mit rund 100 Studierenden aus 34 Ländern. In Verbindung mit dem Zeltlager wurde ein wissenschaftlicher Kongress durchgeführt. Der Kongress wurde von Prof. Dr. Krümmel und Reichssportführer von Tschammer und Osten eröffnet. Deutsche Referenten waren u.a. Prof. Dr. Möckelmann und Prof. Dr. Jaeck. Sie berichteten ihren Zuhörern über biologische, physiologische und anthropometrische Messungen bei Athleten. Nach den Berichten in der Zeitschrift „Politische Leibeserziehung“, Nr. 8, die sich mit den Olympischen Spielen beschäftigte, gab es keine nationalsozialistischen Indoktrinationen für die ausländischen Studierenden im Rahmen des wissenschaftlichen Kongresses. Krümmel formulierte einleitend in seiner Eröffnungsrede: 
„Es ist daher die große Aufgabe der Führer und Lehrer der körperlichen Erziehung in allen Ländern dafür Sorge zu tragen, daß die Jugend im olympischen Geist erzogen wird, im Geiste des friedlichen Wettkampfs“ (Krümmel, 1936, (8), S. 5).

Anlässlich der Olympischen Spiele von Berlin wurde auch eine Reihe von Broschüren und Heften gedruckt, um Kinder und Jugendliche in deutschen Schulen mit der historischen Entwicklung der Olympischen Bewegung und ihren Spielen vertraut zu machen und die verschiedenen olympischen Sportarten mit ihren Rekorden vorzustellen.

Nach dem 2. Weltkrieg tauchte der Gedanke, die Olympischen Spiele mit dem Jugendsport und der Leibeserziehung in den Schulen zu verbinden, bereits 1948 anlässlich der Olympischen Spiele von London erneut auf. Der damalige Vizepräsident des US-amerikanischen Olympischen Komitees (USOC) Wilson äußerte sich dazu:

"I think the most appalling failure is our lack of Olympic education to the youth of America and a most valuable work of Olympic education could be promulgated" (Psimopoulos, 2014, S. 208; Psimopoulos et al., 2017, S. 293).

In Helsinki 1952 gab es ein Lager der Deutschen Sportjugend mit 400 Teilnehmern und bei den Olympischen Spielen 1956 in Melbourne wurde erstmals ein Weltkongress für Leibeserziehung veranstaltet. Über 350 international bekannte sportpädagogische Fachkräfte aus 26 Ländern, einige auch aus Deutschland, diskutierten über mehrere Tage die Entwicklung der Leibeserziehung und hatten schließlich die Idee, ein globales Netzwerk für Sportwissenschaftler und Sportpädagogen als Weltrat zu gründen.

Wie Stephen Bailey (1996) in seiner Geschichte über den Weltrat ausführt, verabschiedete der Kongress in Melbourne die geplante Gründung des Weltrates für Sport und Leibeserziehung (ICSPE) als

"one international physical education body which might be accepted by the United Nations as a body with consultant status to advice on all matters relating to physical education, and to investigate world problems in physical education" (Bailey, 1996, S. 38).

Vier Jahre später, anlässlich der Olympischen Spiele von Rom, gründete sich dann aus diesem Netzwerk heraus der „International Council of Sport and Physical Education“ (ICSPE) (Bailey, 1996, S. 65ff.). Seit dem Jahr 1982 trägt der Weltrat den Zusatz „Sport Science“, um auch den sportwissenschaftlichen Interessen in der Außenwirkung genüge zu tragen und 
wird zum „International Council of Sport Science and Physical Education“ (ICSSPE) (Bailey, 1996, S.3f.).

Auch das vormalige internationale Jugendcamp bei Olympischen Spielen wurde vom Organisationskomitee der Olympischen Spiele von Tokio 1964 wieder neu aufgelegt. Die nationalen Erziehungsbehörden in Japan und das örtliche Organisationskomitee in Tokio gingen sogar noch einen Schritt weiter. Gemeinsam erarbeiteten und veröffentlichten sie mehrere Broschüren, Hefte und Poster, um die Olympische Idee und die Geschichte der Olympischen Spiele den japanischen Schülern und Schülerinnen landesweit näher zu bringen.

Taro Obayashi (2017) dokumentiert sehr detailliert die Vorbereitung und Durchführung dieser frühen olympischen Erziehungskampagne. Sie hatte sieben Säulen:

"Understanding the Olympics, international understandings, public morality, commercial morality, traffic morality, beautification of health promotion" (Obayashi, 2017, S. 39).

Mit den Olympischen Spielen von München 1972 sind in dieser Hinsicht ebenfalls zwei besondere Merkmale verbunden. Zum einen der erste olympische Wissenschaftskongress seiner Art vor Beginn der Eröffnung von Olympischen Spielen (Baitsch et al., 1972) und zum anderen Aktivitäten des Bayerischen Kultusministeriums schon im Schuljahr vor den Spielen, mit dem Ziel, das Thema Olympia im Schulunterricht aufzugreifen. Der stellvertretende Staatssekretär Erwin Lauerbach referierte u.a. auf der 12. Session der Internationalen Olympischen Akademie in Olympia im Frühjahr 1972 zum Thema: „The Propagation of Olympic Principles at Schools“ (Lauerbach, 1973). Aus Anlass der Münchener Spiele veröffentlichte die Deutsche Olympische Gesellschaft (DOG) ein Lesebuch mit einer Auswahl von Kurzgeschichten und Gedichten zum Sport, geschrieben von bekannten deutschen Schriftstellern (DOG, 1971).

Der Begriff der Olympischen Erziehung mag im Hintergrund gestanden haben, als Leibeserzieher und Sportpädagogen in verschiedenen Schulprogrammen anlässlich von Olympischen Spielen die Olympische Idee oder die Olympischen Prinzipien Kindern und Jugendlichen näherbringen wollten. Konkret wurde dieser Begriff erstmals durch eine Publikation von Norbert Müller (1975) bekannt und erreichte erst in den 1980er und 1990er Jahren, einerseits über die weitere Entwicklung von Unterrichtsmaterialien, andererseits über Vorträge an der Internationalen Olympischen Akademie in Olympia weltweite Verbreitung. Ein weiterer Schritt zur Förderung der Olympischen Erziehung war die Aufforderung des IOC (1983) an alle Nationalen Olympischen Komitees, eigene Natio- 
nale Olympische Akademien zu gründen, die heute in rund 150 Ländern existieren.

In Deutschland erhielt die Entwicklung der Olympischen Erziehung durch die Broschüren und Unterrichtshefte für 6- bis 12-jährige Schulkinder aus Anlass der Olympischen Spiele durch das NOK ab 1988 und später durch die Deutsche Olympische Akademie (DOA) wichtige Impulse, die durch die sportpädagogische Fachdiskussion ab den 1990er Jahren verstärkt wurden (Geßmann 1992, 2004; Grupe 1997; Müller 1998; Naul, 2002, 2007; Naul et al., 2008). Allerdings gibt es bis heute keine Grundlagenforschung über die Olympischen Kongresse, um ihre Funktion für die Traditionsbildung (Legacy) für Bildung, Sport, Gesundheit und Sozialarbeit zu verorten (Naul, et al., 2019). In den Worten von Coubertin heißt das "... as yet [not done, Anm. d. Verf.] - it must be" (Coubertin, 1918, in Müller, 2000, S. 217).

\section{Literatur:}

Bailey, S. (1996). Science in the Service of Physical Education and Sport. London: Wiley.

Baitsch, H., Bock, W. E., Bolte, M., Bokler, W., Grupe, O., Heidland, H. W. \& Lotz, F. (1972). Sport im Blickpunkt der Wissenschaften. Berlin/Heidelberg/New York: Springer.

Comité International Olympique. (1908). Annuaire, Règlement, But. o.O.

Coubertin, P. de (1922). La pedagogie sportive. Paris: Cres.

Coubertin, P. de (1928). Die sportliche Erziehung. Stuttgart: Enke.

Coubertin, P. de (1936). Olympische Erinnerungen. Berlin: Limpert.

Da Costa, L. (2002). Olympic Studies. Rio de Janeiro: University of Gama de Filho.

Deutsche Olympische Gesellschaft (Hrsg.) (1971). Olympisches Lesebuch. Hannover: Schroedel.

Gebhardt, W. (1904). Die olympische Bewegung und die Schule. In P. Schubert (Hrsg.), Bericht über den I. Internationalen Kongress für Schulhygiene, III, (S. 109 116). Nürnberg: Schrag.

Georgiadis, K. (1995). International Olympic Academy: The history of its establishment, aims and activities. In IOA (Hrsg.), $2^{\text {nd }}$ Joint International Session for Directors of National Olympic Academies, Members and Staff of National Olympic Committees and International Sport Federations (S. 15 - 21). Athens: IOA.

Geßmann, R. (1992). Fachdidaktische Ansätze zur olympischen Erziehung in der Schule - Versuch einer Bestandsaufnahme. Sportunterricht, 41, 193-200.

Geßmann, R. (2004). Olympisches Menschenbild und schulische Sportdidaktik. In NOK (Hrsg.), Olympische Erziehung. Eine Herausforderung an Sportpädagogik und Schulsport (S. 131 - 153). St. Augustin: Academia. 
Grupe, O. (1997). Olympisches Menschenbild und olympische Erziehung. Abschied von einer großen Idee? In O. Grupe (Hrsg.), Olympischer Sport Rückblick und Perspektiven (S. 223-243). Schorndorf: Hofmann.

Guth, J. (1896). Die olympischen Spiele in Athen 1896. Zeitschrift für die österreichischen Gymnasien, 11, 961-975.

Hamer, E. U. (1971). Willibald Gebhardt 1861-1921. Köln: Barz \& Beienburg.

Huhn, K. (1992). Der vergessene Olympier. Das erstaunliche Leben des Dr. Willibald Gebhardt. Berlin: Spotless.

Kemény, F. (1897). Die Bedeutung der Olympischen Spiele für die körperliche Erziehung der Jugend. Zeitschrift für das Realschulwesen, 22, 129-141,195-206.

Krüger, A. (2009). Die Olympische Erziehung im Spätwerk Coubertins. In R. Naul, A. Krüger, \& W. Schmidt (Hrsg.) Kulturen des Jugendsports - Bildung, Erziehung und Gesundheit (S. 15 - 38). Aachen: Meyer \& Meyer.

Krüger, M. (2018). Sport und Olympische Erziehung. In A. Güllich \& M. Krüger (Hrsg.), Sport in Kultur und Gesellschaft. Berlin: Springer https://doi.org/10.1007/ 978-3-662-53385-7_26-1.

Krümmel, C. (1936) Sportstudenten aus aller Welt. Politische Leibeserziehung, 3 (8), 5-8.

Landry, F. (1980). The Games of the XXIst Olympiad and the Promotion of Olympism in Quebec Schools. In IOA (Hrsg.) Report of the International Session for Educationists 1973-1977-1980 (S. 287-298). Athens: HOC.

Lämmer, M. (1982/83). Der sogenannte Olympische Friede in der griechischen Antike. Stadion 8/9, S. 47-83.

Lauerbach, E. (1973). The propagation of Olympic principles in school. In HOC (Hrsg.), Report of the 12th Session of the IOA (S. 98-107). Athens: IOA.

Lennartz, K. (1978). Die VI. Olympischen Spiele Berlin 1916. Köln: Bartz \& Beienburg.

MacAloon, J. J. (2007). This Great Symbol: Pierre de Coubertin and the Origins of the Modern Olympic Games. London: Routledge.

Müller, N. (1975). Olympische Erziehung. In F. Thaller \& H. Recla (Hrsg.), Signale der Zeit (S. 133 - 140). Schorndorf: Hofmann.

Müller, N. (1977). The Olympic idea of Pierre de Coubertin and Carl Diem and its materialisation in the International Olympic Academy. In HOC (Hrsg.), Report of the 16th Session of the IOA (S. 94 - 100). Athens: IOA.

Müller, N. (1994). One hundred years of Olympic Congresses, 1894-1994. Niedernhausen: Schors.

Müller, N. (1995). Olympia zwischen Idealität und Realität. Die Internationale Olympische Akademie im Spiegel der Vorträge 1961-1994. Niedernhausen: Schors.

Müller, N. (1998). Olympische Erziehung. In O. Grupe \& D. Mieth (Hrsg.), Lexikon der Ethik im Sport (S. 385-395). Schorndorf: Hofmann.

Müller, N. (2000). Pierre de Coubertin. Selected Writings. Lausanne: IOC. 
Naul, R. (1989). Gymnastics, athletics, games: Sedan celebrations in imperial Germany. In M. Lämmer (Hrsg.), Proceedings of the XII HISPA-Congress (S. 131 - 136). St. Augustin: Academia.

Naul, R. (1999). Willibald Gebhardt - Leben und Werk des Pioniers der internationalen Olympischen Bewegung in Deutschland. In R. Naul \& M. Lämmer (Hrsg.), Willibald Gebhardt - Pionier der Olympischen Bewegung (S. 11 - 29). Aachen: Meyer \& Meyer.

Naul, R. (2002). Olympische Erziehung - Chancen und Aufgaben für den Schulsport. Sportunterricht, 51, 267-272.

Naul, R. (2007). Olympische Erziehung. Aachen: Meyer \& Meyer.

Naul, R. (2010). Olympic Education. (2 ${ }^{\text {nd }}$ ed.), Maidenhead: Meyer \& Meyer.

Naul, R., Geßmann, R. \& Wick, U. (2008). Olympische Erziehung in Schule und Verein. Grundlagen und Materialien. Schorndorf: Hofmann.

Naul, R., Krüger, M., Hübner, E., Georgiadis, K., Szikora, K., Skoda, Z. \& Wassong, St. (2019). Forgotten Olympic legacies: the impact of the early Olympic Games and Olympic Congresses on education, sport, health, and social work and its revival as Olympic education after the Olympic Centennial Congress 1994. (Paper presented at the UPEM Congress "L'heritage des Jeux Olympiques et Paralympiques”, Paris, Juin, 2019).

Obayashi, T. (2017). Tokyo 1964: Nation-wide Olympic education programme for Tokyo 1964 initiated by the Japanese Government. In R. Naul, D. Binder, A. Rychtecky, \& I. Culpan (Hrsg.), Olympic education - an international review (S. 35 - 46.) London: Routledge.

Psimopoulos, C. S. (2014). Teaching Olympic Education in the USA. In D. Chatziefstathiou \& N. Müller (Hrsg.), Olympism, Olympic Education and Learning Legacies (S. 207-218). New Castle upon Tyne: Cambridge Scholars.

Psimopoulos, C. S., Binder, D. Vermillon, A. \& Naul, R. (2017). United States of America: The Olympic Games as promotor of Olympic education: issues and institutions. In R. Naul, D. Binder, A. Rychtecky, \& I. Culpan (Hrsg.), Olympic education - An international review (S. 291-304). London: Routledge.

Wassong. S. (2002). Pierre de Coubertins US-amerikanische Studien und ihre Bedeutung für die Analyse seiner früheren Erziehungskampagne. Würzburg: Ergon. 
